\title{
Dinámicas socioecológicas y valores sociales de servicios ecosistémicos en la cuenca del río Savegre, Costa Rica
}

\section{Social-ecological Dynamics and Social Values of Ecosystem Services in the Savegre River Watershed, Costa Rica}

\author{
Edgar Espinoza Cisneros* (1) https://orcid.org/0000-0002-1018-3440 \\ Linnette Ceciliano Calderón ** (1) https://orcid.org/0000-0001-7593-9299
}

\begin{abstract}
Resumen
Objetivo: examinar las dinámicas socioecológicas que explican las configuraciones espaciales de valores sociales sobre servicios ecosistémicos en la cuenca del río Savegre, Costa Rica. Metodología: se usaron un mapeo participativo, encuestas y entrevistas abiertas aplicadas a una muestra de 95 administradores de unidades productivas en la cuenca. Resultados: se encontró que, para este sistema, la zona de transición entre la cuenca alta y la cuenca media es importante en su concentración de valores sociales sobre servicios ecosistémicos. El valor social de biodiversidad fue muy priorizado y generalizado en términos espaciales. Por el contrario, otros valores sociales más abstractos, como el valor futuro y el estético, muestran tendencias más locales. Además, las transiciones históricas de usos de la tierra, así como factores biofísicos, como la topografía y eventos climáticos, se conjugan para determinar las configuraciones espaciales de los valores sociales. Limitaciones: la representación cartográfica de los valores sociales se basó en el uso de puntos debido a las características del programa utilizado (SolVES), por lo que no refleja asignaciones espaciales de valores sociales que comprenden la totalidad de la cuenca o un sector de la misma. Valor y conclusiones: se busca proveer insumos para el diseño de estrategias de sostenibilidad, sustentadas en evaluaciones socioambientales que trasciendan lo pecuniario e incorporen criterios socioculturales explícitos en términos espaciales.

Palabras clave: mapeo participativo; cogniciones ambientales; interacciones ser humano/ambiente; valores ambientales; río Savegre.
\end{abstract}

\begin{abstract}
Objective: This paper examines some socio-ecological dynamics that explain the spatial configurations of ecosystem services social values the Savegre river watershed in Costa Rica. Methodology: participatory mapping, surveys and open interviews applied to a sample of 95 land managers were used. Results: it was found that the transition zone between the upper and the middle sections of the watershed is particularly important for this system, due its concentration of social values of ecosystem services. The social value of biodiversity was highly prioritized and generalized in spatial terms. In contrast, other more abstract social values, such as future and aesthetic values, show more local tendencies. In addition, historical land use transitions, as well as biophysical factors, like topography and climatic events, conjugate to determine spatial configurations of social values. Limitations: the cartographic representation of social values was based on the use of point features due to the characteristics of the program used (SolVES); therefore, it does not reflect spatial allocations of social values that cover the whole watershed or a sector of it. Value and conclusion: the principal aim is to provide insights for the design of sustainability strategies, based on socio-environmental assessments that transcend the exclusively pecuniary and incorporate spatially-explicit socio-cultural criteria.

Keywords: participatory mapping; environmental cognitions; human/environment interactions; environmental values; Savegre River.
\end{abstract}

Cómo citar: Espinoza Cisneros, E., y Ceciliano Calderón, L. (202I). Dinámicas socioecológicas y valores sociales de servicios ecosistémicos en la cuenca del río Savegre, Costa Rica. región y sociedad, 33, el4I8. doi: I0.22 I98/rys202I/33//4I8

* Autor para correspondencia. Universidad de Costa Rica, Escuela de Geografía. Montes de Oca Núm. 2060, San José, Costa Rica. Correo electrónico: edgar.espinoza@ucr.ac.cr

** Universidad de Costa Rica, Escuela de Geografía. Montes de Oca Núm. 2060, San José, Costa Rica. Correo electrónico: lincalde21@gmail.com

Recibido: 21 de noviembre de 2020

Aceptado: 13 de abril de 2021

Liberado: 3 de mayo de 2021

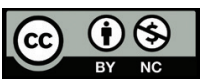




\section{Introducción}

Ante los crecientes retos en la gestión ambiental, la atención reciente se ha dirigido a comprender mejor los factores psicosociales que subyacen las decisiones y acciones humanas sobre el ambiente. De manera creciente se han incorporado tales consideraciones en evaluaciones ambientales, con el propósito de diseñar mejores estrategias e intervenciones para sistemas socioecológicos más resilientes (Arias-Arévalo, Martín-López y Gómez-Baggethun, 2017; Brondizio y Gatzweiler, 2010; Jacobs, Dendoncker, Martín-López, Barton, Gómez-Baggethun, Boeraeve, McGrath, Vierikko, Geneletti, Sevecke, 2016; Jones, Shaw, Ross, Witt y Pinner, 2016).

En particular, los valores han recibido más atención en estudios ambientales y en las ciencias sociales en general (Jacobs et al., 2016; Tadaki, Sinner y Chan, 2017; West, Haider, Masterson, Enqvist, Svedin y Tengö, 2018), dado que constituyen la base sobre la cual se yerguen los demás constructos cognitivos, como las creencias, las actitudes, las normas, las intenciones y, finalmente, las conductas (Ives y Kendal, 2014; Larson, 2010). Existen múltiples interpretaciones y conceptualizaciones de los valores, pero en general éstas se bifurcan en valores subyacentes (held values) y valores asignados (assigned values) (véase tabla 1). Por lo general usados en plural, los primeros son los valores primordiales, pues guían criterios en torno a lo que se considera apropiado mientras que los segundos, que casi siempre se emplean en singular, se refieren a la valoración o magnitud de preferencia que se tiene de un objeto o lugar específico y que puede o no ser de carácter monetario. La primera conceptualización está muy asociada con disciplinas como la filosofía y la psicología, mientras que la segunda está más vinculada a la economía. Relacionando ambos tipos de valores en el contexto ambiental, los subyacentes representan los cimientos sobre los cuales se construyen los asignados con las interacciones entre ser humano y medio ambiente como el proceso mediador entre ambos (véase figura 1). En efecto, en dichas interacciones ambos tipos de valores son importantes por su significativa influencia en los procesos de toma de decisiones (Dietz, Fitzgerald y Shwom, 2005).

Los valores asignados son importantes para evaluaciones ambientales, ya que manifiestan "cómo la gente piensa sobre aspectos específicos del ambiente, incluyendo la importancia que le dan a ciertos lugares, especies o funciones ecológicas” (Jones et al., 2016, p. 5. Traducción propia). Los valores sociales forman parte de los valores asignados, pues reflejan preferencias hacia ciertos objetos o lugares y son por lo común asociados con un contexto espacial determinado. Por eso en términos espaciales son útiles para entender la relación entre cogniciones ambientales ${ }^{1}$ y espacio geográfico (Bryan, Raymond, Crossman y Macdonald, 2010).

Compartimos la definición general de cogniciones ambientales de Meyfroidt (2013, p. 342), como "los elementos cognitivos que se relacionan con el ambiente natural, los recursos naturales y las actividades vinculadas a la naturaleza" (traducción propia). Texto original: "Environmental cognitions are defined here as the cognitive features that relate to natural environment and natural resources and to activities that take place in relation with nature". 
Tabla 1. Algunas características diferenciadoras entre los valores subyacentes y los valores asignados

\begin{tabular}{|c|c|}
\hline Valores subyacentes & Valores asignados \\
\hline Más estables y lentos en cambiar & Mayor plasticidad y cambian más rápido \\
\hline Más generales y universales & $\begin{array}{c}\text { Son más contexto-dependientes; } \\
\text { por ende, son asociados con objetos, } \\
\text { lugares o elementos naturales específicos }\end{array}$ \\
\hline $\begin{array}{c}\text { Representan ideales más } \\
\text { primordiales sobre lo que } \\
\text { "debería ser" }\end{array}$ & $\begin{array}{c}\text { Representan preferencias específicas } \\
\text { sobre el valor de un objeto o lugar }\end{array}$ \\
\hline $\begin{array}{c}\text { Más utilizados en campos } \\
\text { disciplinarios como la filosofía } \\
\text { y psicología }\end{array}$ & $\begin{array}{c}\text { Más utilizados en campos } \\
\text { como la economía }\end{array}$ \\
\hline $\begin{array}{c}\text { Están en la base de la jerarquía } \\
\text { cognitiva; subyacen otros constructos, } \\
\text { como actitudes, creencias y normas }\end{array}$ & $\begin{array}{c}\text { Están más arriba en la jerarquía cognitiva; } \\
\text { están más asociados de manera directa } \\
\text { con conductas específicas }\end{array}$ \\
\hline
\end{tabular}

Fuente: elaboración propia.

Figura 1. Esquema conceptual sobre las relaciones entre los valores subyacentes y valores asignados en un marco de interacciones ser humano/ambiente

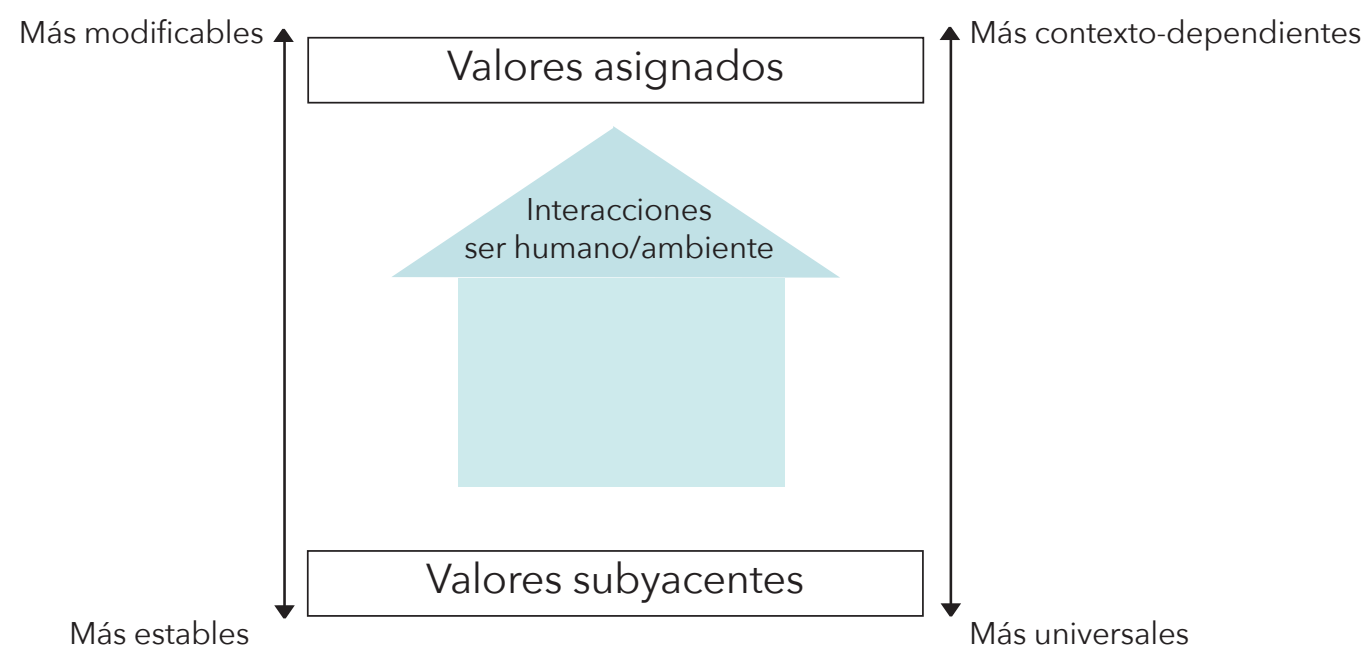

Fuente: adaptado de Van Riper y Kyle (2014, p. 375). 
En geografía y disciplinas afines se ha profundizado en el estudio de las dimensiones espaciales de los factores cognitivos, entre éstos los valores sociales. Evidencia de lo anterior es la aparición de herramientas geoespaciales incorporadas en sistemas de información geográfica (SIG) (Ives y Kendal, 2014). Los sistemas de información geográfica participativos (SIGP), por ejemplo, han contribuido a la espacialización de estos componentes cognitivos otrora ignorados en la planificación territorial, y en la incorporación de más participantes de la sociedad civil en procesos de planificación de carácter geoespacial (Barrera Lobatón, 2009; Brown y Kyttä, 2014). Varios trabajos ya han tratado la teoría, la metodología y los aspectos empíricos asociados con la espacialización de los valores sociales (Sherrouse, Semmens y Clement, 2014; Ives y Kendal, 2014; Bryan et al., 2010; Van Riper y Kyle, 2014; Brown y Fagerholm, 2015), por lo que no se ahondará en esos detalles aquí.

Las métricas del paisaje social (social landscape metrics) constituyen otro abordaje empírico resultante de esta creciente popularidad de las dimensiones espaciales de los valores. Estas métricas surgen como contraparte a las que suelen utilizarse en la ecología del paisaje para cuantificar la estructura, distribución y composición de los elementos físicos de éste, como parches boscosos, fauna y flora o patrones de asentamientos humanos (Vila Subirós, Linde, Llausàs Pascual y Ribas Palom, 2006). Las métricas del paisaje social, por el contrario, se enfocan en cuantificar y analizar la distribución y estructura en el espacio de percepciones, valores, actitudes y otras cogniciones humanas que se relacionan con el lugar (Brown y Reed, 2012). Así, con el fin de brindar mejores insumos para la planificación territorial, se han concebido metodologías y conceptos para entender mejor la relación dinámica entre cogniciones y espacio geográfico (Nassauer, 1995).

El constructo de los valores sociales se ha aplicado al campo de los servicios ecosistémicos, un paradigma que ha logrado articular las dinámicas ecológicas con las sociales bajo la sombrilla del bienestar humano. Simplificando, los servicios ecosistémicos representan las contribuciones tanto directas como indirectas de la naturaleza al bienestar humano, y comprenden desde los alimentos y el agua para el sustento material hasta los servicios culturales intangibles como la espiritualidad y la apreciación estética (Intergovernmental Science-Policy Platform on Biodiversity and Ecosystem Services [IPBES], 2019; Millennium Ecosystem Assessment [MEA], 2005). Esos servicios derivan de procesos y funciones ecológicas, y a su vez el ser humano los valora de diversas maneras, dependiendo, entre otras cosas, de las percepciones sobre su contribución al bienestar (véase figura 2). En el contexto de los servicios ecosistémicos, en la presente investigación se entienden los valores sociales como las atribuciones individuales y colectivas de valor, por lo general no monetario, a las cualidades de los ecosistemas (funciones, procesos y servicios) y los beneficios que éstos proveen al bienestar humano (Codato, 2016; Sherrouse y Semmens, 2015; Van Riper, Kyle, Sutton, Barnes y Sherrouse, 2012). 
Figura 2. Diagrama basado en el modelo de "cascada" de los servicios ecosistémicos (Potschin-Young, Haines-Young, Görg, Heink, Jax y Schleyer, 2018)

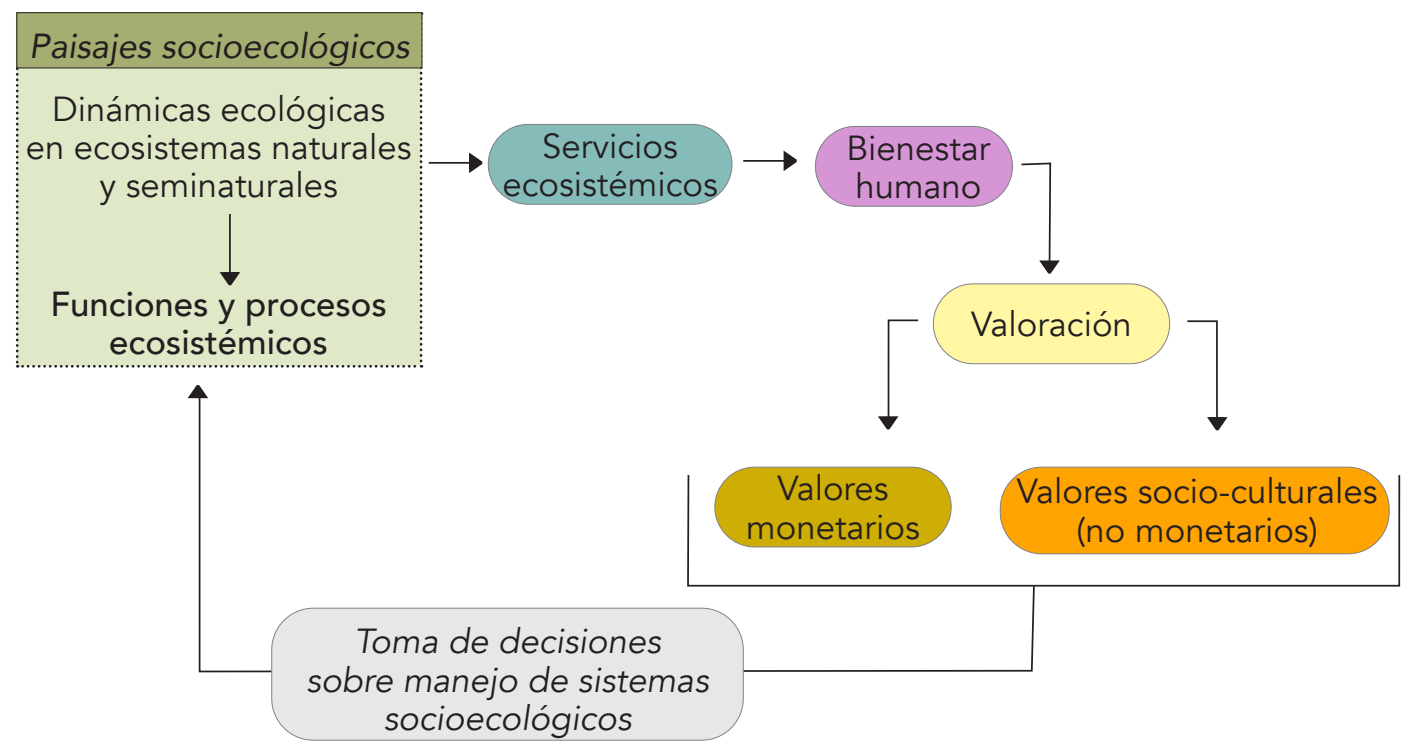

Fuente: adaptado de Martín-López, Gómez-Baggethun, García-Llorente y Montes (2014, p. 222).

Numerosos estudios han explorado la distribución espacial de los valores sociales sobre servicios ecosistémicos en diversos sistemas socioecológicos (Bagstad, Semmens, Ancona y Sherrouse, 2017; Codato, 2016; De Vreese, Leys, Fontaine y Dendoncker, 2016; Sherrouse Semmens y Clement, 2014; Sun, Xiang, Tao, Tong y Che, 2019), muchos utilizando herramientas como los SIGP (Brown, 2013; Brown y Fagerholm, 2015; García-Nieto, Quintas-Soriano, García-Llorente, Palomo, Montes y Martín-López, 2015; Johnson, Riper, Chu y Winkler-Schor, 2019). Sin embargo, pocos han examinado en profundidad el porqué de esas distribuciones espaciales valiéndose de métodos cualitativos que complementen los ejercicios de mapeo participativo. En este artículo se analizan las dinámicas socioecológicas ${ }^{2}$ que ayudan a explicar las configuraciones espaciales de los valores sociales sobre servicios ecosistémicos que provee un sistema de cuenca fluvial en Costa Rica. Con este fin, se complementa en términos metodológicos la cartografía resultante de los procesos participativos con los insumos cualitativos de entrevistas y encuestas. Así, se busca contribuir al entendimiento de las dimensiones espaciales de los valores sociales y sobre todo de la relación que se da entre el contexto socioecológico y las cogniciones ambientales en zonas rurales.

2 Por dinámicas socioecológicas nos referimos a los procesos complejos producto de las interacciones, a diversas escalas espaciales y temporales, entre los subsistemas sociales y biofísicos que conforman un sistema acoplado ser humano/medio ambiente. 


\section{Métodos}

\section{Sitio de estudio}

La cuenca del río Savegre se ubica en la región Pacífico Central de Costa Rica (véase figura 3). Su extensión total es de $590 \mathrm{~km}^{2}$, aproximadamente $1.15 \%$ del territorio costarricense. La cuenca es reconocida a escala nacional e internacional como un lugar de atractivo ecoturístico, de ríos limpios y como una "zona caliente" de biodiversidad y servicios ecosistémicos (Acevedo, Bustamante, Paniagua y Chaves, 2002; Ministerio de Ambiente y Energía [MINAE], 2004). La cuenca se distingue por presentar parches de bosque natural continuo desde la divisoria continental hasta la costa. Cerca de $75 \%$ del área de la cuenca se encuentra bajo cobertura forestal, mucha de la cual está bajo algún régimen formal de protección. En 2017, el área de la cuenca fue designada por la UNESCO como reserva de la biosfera, en reconocimiento a su alto valor ecológico, su importancia socioeconómica y el potencial para "probar abordajes interdisciplinarios para el entendimiento y el manejo de los cambios e interacciones entre sistemas sociales y ecológicos" (UNESCO, 2017).

Figura 3. Mapa de la cuenca hidrográfica del río Savegre

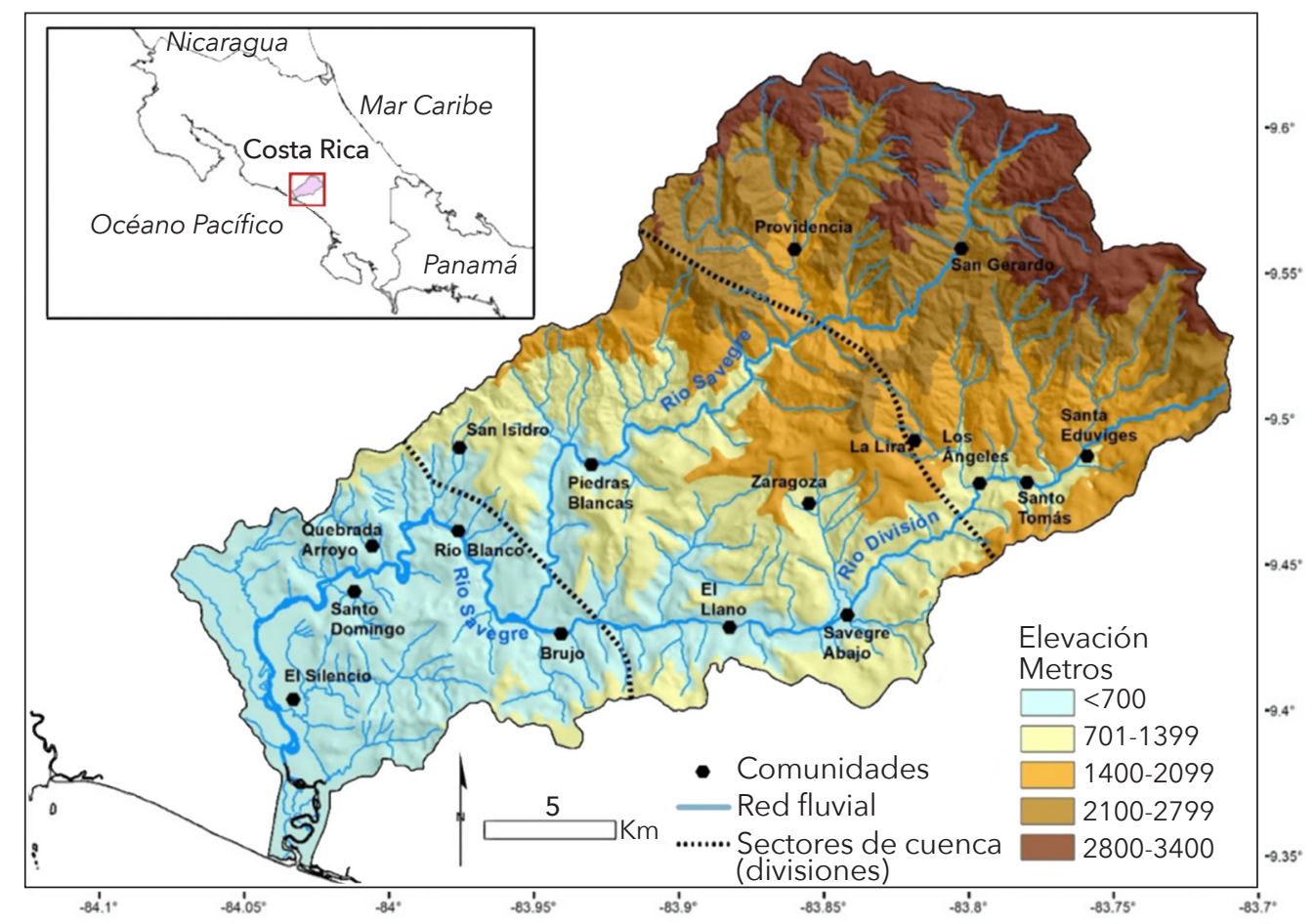

Fuente: elaboración propia (2020). 
Si bien no existen datos confiables y recientes sobre la población de la cuenca, la densidad poblacional se estima en 30 personas por km² (Mattey, 2014), una tercera parte de la cifra a nivel de país. Esta baja densidad poblacional se debe en parte a la abrupta y pronunciada topografía de la cuenca, la cual ha indispuesto los asentamientos humanos, sobre todo en las zonas altas y medias. Las principales actividades productivas son la agricultura (café, palma africana y frutas como aguacate, moras y manzanas), la ganadería y el ecoturismo. Muchas unidades productivas en la cuenca son sistemas mixtos de las actividades antes mencionadas, pero sobre todo de agricultura y ganadería, y en menor grado, de turismo.

La cuenca del Savegre se caracteriza por una “contradicción” entre narrativas y degradación ambiental que hace importante aquí un análisis espacial de las dimensiones socioculturales de los servicios ecosistémicos. Por un lado, se la conoce como una cuenca muy conservada, biodiversa y de ríos limpios, así como también de comunidades muy responsables del medio ambiente (Espinoza-Cisneros y Akhter, 2020; Gobierno de Costa Rica, 2015). Por otro lado, sin embargo, se ha visto expuesta en las últimas décadas a presiones antropogénicas producto de la acuicultura intensiva, de la expansión de las fronteras agrícolas y ganaderas y de la pesca ilegal con técnicas destructivas (Espinoza-Cisneros, 2018; Naranjo, 2016; Sistema Nacional de Áreas de Conservación [SINAC], 2017). A la luz de estas problemáticas, se han conformado grupos intercomunitarios e incluso a nivel de cuenca con el fin de minimizar los impactos en la integridad ecológica del sistema y fomentar la producción sostenible.

Recolección de datos

Para recolectar los datos sobre valores sociales, se llevó a cabo un ejercicio de mapeo participativo complementado con encuestas y entrevistas presenciales aplicadas a 95 administradores de unidades productivas a lo largo de la cuenca del río Savegre (36 en la cuenca alta, 26 en la media y 33 en la baja). Se hizo el ejercicio con los administradores de unidades productivas debido a que son los principales agentes tomadores de decisiones a este nivel, cuyas resoluciones determinan en gran medida las transformaciones del paisaje socioecológico de la cuenca. La recolección de datos se realizó entre mayo de 2016 y marzo de 2018 en 16 comunidades de la cuenca. El mapeo participativo consiste en un proceso colectivo e inclusivo de recolección, procesamiento, análisis y divulgación de datos e información de naturaleza geoespacial para la toma de decisiones, sobre todo a escala comunitaria. El diseño de los instrumentos de las encuestas y el mapeo participativo se basó en Clement (2006) y Brown, Reed y Harris (2002). Los administradores fueron elegidos mediante una técnica de muestreo aleatorio estratificado utilizando listas que proveen las asociaciones de desarrollo integral (ADI) de cada comunidad. Comparado con municipalidades e instituciones estatales a escala nacional, las ADI se consideraron mejores fuentes de datos sobre unidades productivas de todas las comunidades debido a que suelen llevar registros precisos y actualizados en un contexto rural como éste. Las listas proveídas contenían información sobre las unidades productivas 
de cada comunidad, la actividad productiva primaria de la unidad e información de contacto del administrador.

El ejercicio de mapeo participativo se realizó de manera presencial. En él, cada participante identificó primero los valores sociales que consideraba más importantes en el contexto socioecológico de la cuenca y luego ponderó cada uno a través de un ejercicio de asignación de puntos. Los valores sociales que al principio se consideraron en este estudio, se muestran en la tabla 2; sin embargo, para el análisis que aquí se presenta, sólo se tomaron en cuenta los cinco valores más relevantes según los resultados obtenidos (véase la "Sección de resultados y discusión"). Después se le pidió a cada administrador que ubicara esos valores sociales relevantes en un mapa simplificado de la cuenca que mostraba su delimitación espacial, la red hidrográfica (dado el enfoque en la cuenca) y sus principales comunidades. Se procuró mantener el mapa simple para facilitar su interpretación, evitando la saturación con capas. Se ejecutaron diez pruebas piloto para probar los instrumentos, incluido el mapa para el ejercicio participativo, y se mejoraron con base en ellas mismas. Como último paso, se realizaron entrevistas de preguntas abiertas con cada participante para profundizar sobre los elementos que éste había marcado en el mapa y en la encuesta.

Tabla 2. Valores sociales de servicios ecosistémicos considerados al principio en este estudio

\begin{tabular}{|l|l|}
\hline Tipo de valor & Descripción \\
\hline Estético & $\begin{array}{l}\text { Valoro este río porque disfruto de la belleza del paisaje, } \\
\text { de los sonidos, vistas, aromas, etcétera. }\end{array}$ \\
\hline $\begin{array}{l}\text { Valor de } \\
\text { biodiversidad }\end{array}$ & $\begin{array}{l}\text { Valoro este río porque provee una variedad } \\
\text { de especies de vida silvestre. }\end{array}$ \\
\hline Valor cultural & $\begin{array}{l}\text { Valoro este río porque representa parte de las tradiciones, } \\
\text { conocimiento y estilo de vida de mis ancestros. }\end{array}$ \\
\hline Valor económico & $\begin{array}{l}\text { Valoro este río porque provee pescado, turismo } \\
\text { yagua para uso humano. }\end{array}$ \\
\hline Valor futuro & $\begin{array}{l}\text { Valoro este río porque permite que futuras generaciones } \\
\text { lo conozcan y lo aprovechen como está hoy. }\end{array}$ \\
\hline Valor histórico & $\begin{array}{l}\text { Valoro este río porque es importante para la historia natural } \\
\text { y humana de mi comunidad, región y país. }\end{array}$ \\
\hline Valor intrínseco & $\begin{array}{l}\text { Valoro este río sólo porque existe, independientemente } \\
\text { de la utilidad que tenga para los humanos. }\end{array}$ \\
\hline $\begin{array}{l}\text { Valor de } \\
\text { aprendizaje }\end{array}$ & $\begin{array}{l}\text { Valoro este río por su contribución a la educación, enseñanza } \\
\text { e investigación científica por parte de niños, jóvenes y adultos. }\end{array}$ \\
\hline $\begin{array}{l}\text { Valor de soporte } \\
\text { de vida }\end{array}$ & $\begin{array}{l}\text { Valoro este río porque regula, preserva, limpia y renueva agua } \\
\text { y con ello la vida que dependen de ella. }\end{array}$ \\
\hline Valor de recreación & $\begin{array}{l}\text { Valoro este río porque me permite disfrutar de actividades recreativas. } \\
\text { Valoro este río porque para mí tiene un significado espiritual } \\
\text { o porque siento reverencia y respeto por él. }\end{array}$ \\
\hline Valor espiritual & Valoro este río porque me hace sentir mejor, física y mentalmente. \\
\hline
\end{tabular}

Fuente: tomado de Sherrouse, Semmens y Clement (2014, p. 69). 
Procesamiento y análisis de datos

Los datos de las encuestas y mapeo participativo se procesaron en un sistema de información geográfica (SIG) para su posterior análisis. Para este fin, se utilizó la herramienta Social Values for Ecosystem Services (SolVES por sus siglas en inglés), la cual asiste en el mapeo, cuantificación y evaluación de los valores sociales atribuidos a los servicios ecosistémicos (Sherrouse y Semmens, 2015). SolVES, en su versión 3.0, funciona como una herramienta que se añade al programa ArcGIS de ESRI. Su principal utilidad es proveer métricas cuantitativas, no monetarias y explícitas en términos espaciales de valores sociales para varios grupos de actores, así como también ejecutar modelos estadísticos de las relaciones entre esas métricas con variables ambientales (Sherrouse, Clement y Semmens, 2011). La función de SolVES que más interesaba para este estudio era el cálculo de un índice de valores sociales de diez puntos, indicativo del nivel de intensidad o magnitud de cada valor social para cada pixel y calculado a partir de datos espaciales y no espaciales recabados a través de encuestas de preferencia. ${ }^{3}$ Para facilitar su interpretación, este índice fue categorizado en cinco niveles: muy alto (de 8 a 10), alto (de 6 a menos de 8 ), medio (de 4 a menos de 6), bajo (de 2 a menos de 4) y muy bajo (de cero a menos de dos).

Los mapas resultantes del procesamiento de SolVES fueron luego analizados de forma conjunta con la información cualitativa de entrevistas presenciales. Éstas consistieron en preguntas abiertas de seguimiento (follow-up) sobre lo que el o la participante había contestado en los ítems de las encuestas y el ejercicio de mapeo participativo. Los datos fueron codificados para lograr una segmentación y una posterior clasificación que facilitara su análisis. La información de las entrevistas fue complementada con revisiones exhaustivas de literatura sobre la cuenca del Savegre, en particular estudios técnicos, los planes generales de manejo de las áreas protegidas dentro de la cuenca y artículos científicos sobre ésta publicados en revistas arbitradas.

\section{Resultados y discusión}

La espacialidad de los valores sociales en la cuenca del río Savegre

De los valores considerados al principio, los más priorizados por los participantes fueron los de biodiversidad, futuro, estético, soporte de vida y aprendizaje (véase figura 4). Para enfocar mejor el análisis, en esta investigación se consideraron sólo esos cinco valores sociales (marcados con un asterisco en la figura 4). La figura 5 muestra los mapas de la distribución espacial de estos valores basados en el índice de valores sociales computado en SolVES. En esta figura también se incluye un mapa de dichos valores con el fin de identificar "zonas calientes" (hotspots) de valores sociales. Es importante notar que estos mapas

3 Para más información sobre SolVES, véase solves.cr.usgs.gov o bien consúltese Sherrouse y Semmens (2015). 
no reflejan las atribuciones de valores que se asociaron con el sistema de la cuenca como un todo o con partes de ella, sino que muestran los casos donde el valor se asoció con un punto específico dentro de ésta. En otras palabras, si un participante asociaba el valor de biodiversidad con toda el área de la cuenca y no con un lugar en específico (su comunidad, por ejemplo), eso no se reflejaba en SolVES, debido a que el programa no incorpora funciones para tomar en cuenta todo el sector del área de estudio (o partes del área), sino que trabaja sólo con puntos específicos.

Figura 4. Puntuación total de cada valor social luego de las encuestas y del mapeo participativo con los administradores de unidades productivas

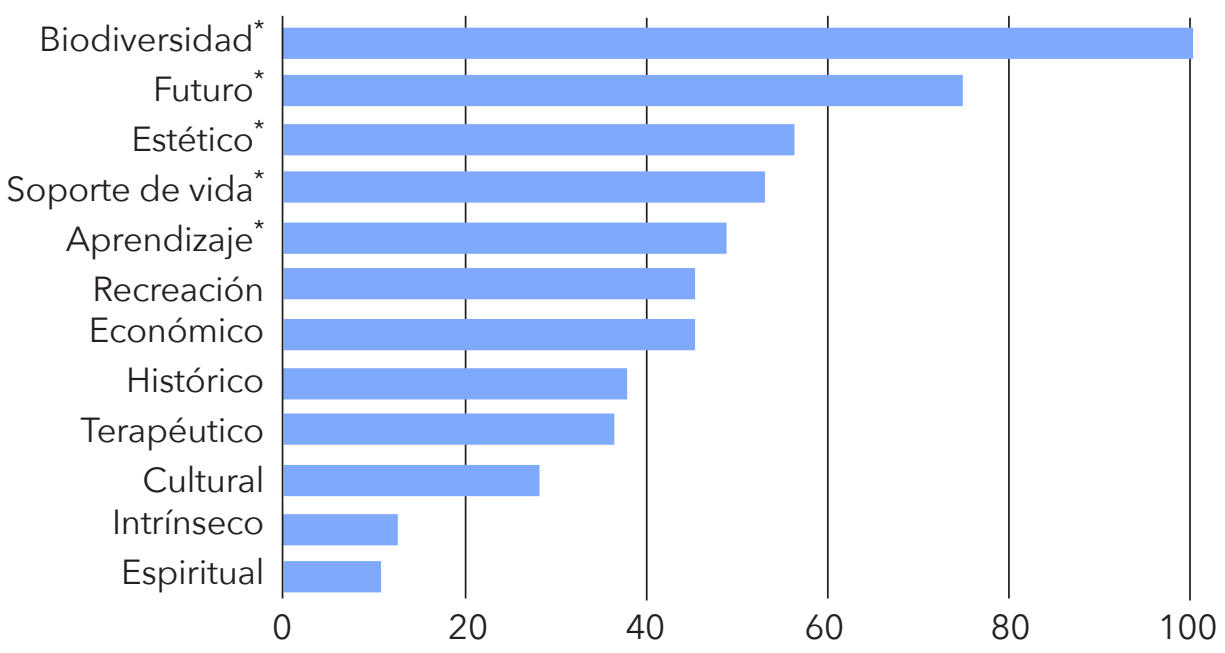

Nota: los valores fueron normalizados a una escala de 100 para facilitar su interpretación. El asterisco marca los cinco valores más priorizados por los participantes y que se consideraron en este estudio.

Fuente: elaboración propia, basado en datos de campo.

Los mapas de la figura 5 muestran que, en general, se dan mayores intensidades de valores sociales en las partes alta y media de la cuenca, en especial en la zona de transición entre esos dos sectores. Dicha zona es importante en cuanto a los valores estético, futuro y soporte de vida. El valor social de biodiversidad es intenso en el sector de la cuenca alta, muy asociado con la ubicación del Parque Nacional Los Quetzales en el extremo noroeste. Resalta también el hecho de que, para este valor social, hubo pocas áreas con niveles de muy bajo (véase figura 5), lo cual es un indicativo importante de los niveles de concientización ecológica de este segmento de población. Por otra parte, el valor de aprendizaje presenta sólo tres niveles: muy bajo, bajo y medio. En este caso, el nivel de medio está más distribuido entre los sectores de la cuenca alta y media. 
Como se indicó antes, el valor estético resalta por presentar los valores de mayor intensidad a lo largo de la zona de transición entre la cuenca alta y la media, con particular concentración en los centros con mayor densidad poblacional hacia el sector este de la cuenca. De manera similar, el valor futuro muestra mayores intensidades a lo largo de la zona transicional entre la cuenca media y la alta, pero con la diferencia de tener más cobertura espacial de los niveles medio y alto. El valor de soporte de vida también muestra una concentración alta cerca de esta misma zona transicional, pero en este caso destaca la categoría de muy bajo en casi todo el sector de la cuenca baja.

El mapa agregado (recuadro rojo en la figura 5) corrobora que, en efecto, la franja transicional entre los sectores de la cuenca alta y la cuenca media concentra los mayores niveles de valores sociales atribuidos a los servicios ecosistémicos, en especial cerca de los centros con mayor densidad poblacional. Por el contrario, la zona de la cuenca baja se caracteriza por las bajas intensidades

Figura 5. Mapas de la distribución espacial de las intensidades de los cinco principales valores sociales en la cuenca del Savegre, según el índice de valores sociales de SolVES
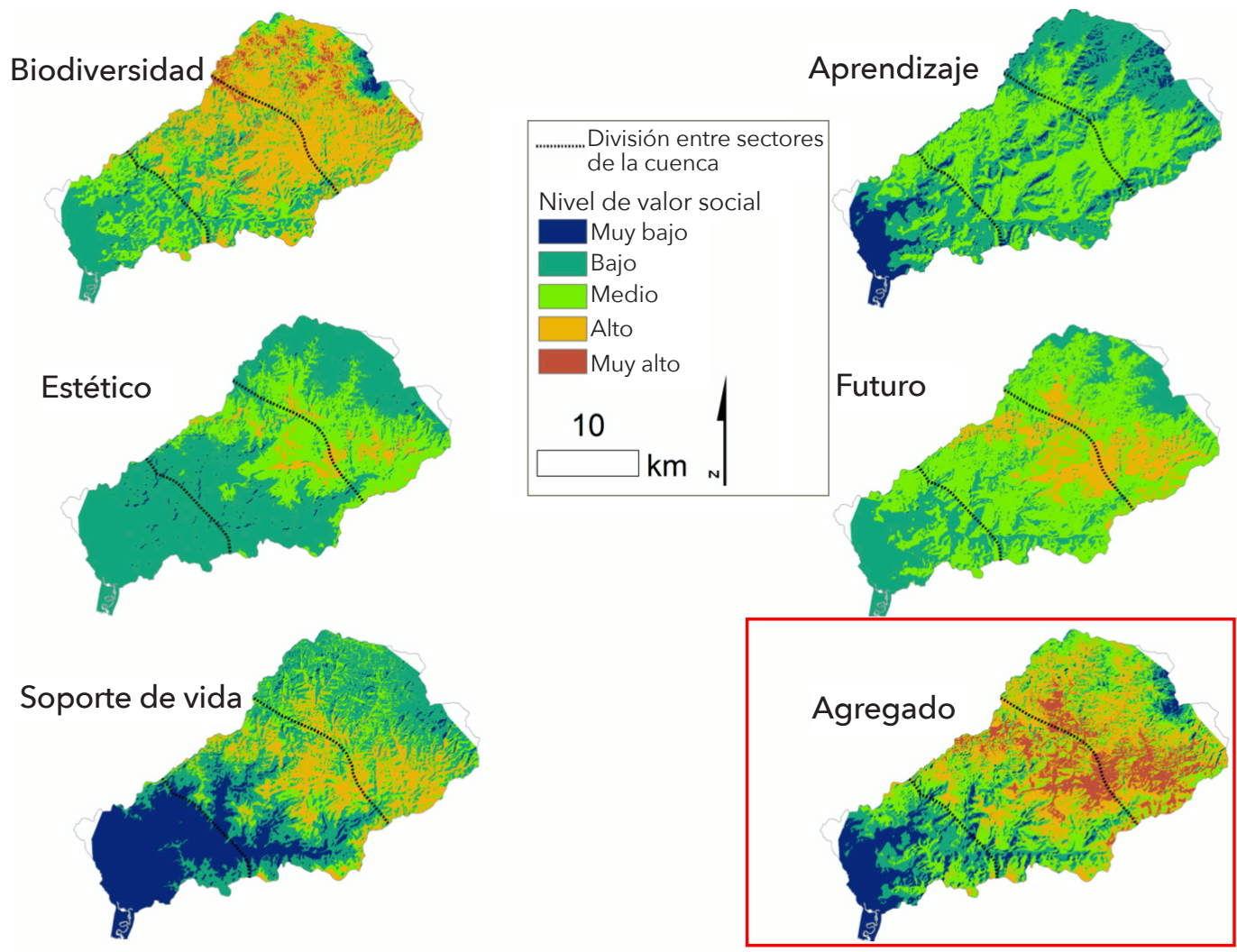

Nota: el mapa en el recuadro rojo muestra los niveles agregados para el principal conjunto de valores sociales.

Fuente: elaboración propia. 
de valores sociales atribuidas. A continuación, se presenta un análisis de estos resultados teniendo en cuenta los insumos de los ejercicios más cualitativos.

Interdependencias funcionales versus localismos

Si bien no se representó en los mapas de valores sociales (véase la "Sección de métodos"), la visión de la cuenca como un sistema socio-hidrológico interconectado y funcionalmente interdependiente resaltó entre los participantes a la hora de espacializar los valores sociales. Del total de participantes que priorizaron el valor de la biodiversidad (60\% del total), $28 \%$ lo asoció de forma espacial con toda la cuenca, mientras que $40 \%$ con sectores de ésta (alta, media, baja) o subcuencas. Esto no es trivial, ya que esta concepción espacial de sistemas integrados e interconectados es parte importante de los modelos mentales necesarios para fomentar una mayor sensibilidad ambiental (Jones et al., 2016; Meyfroidt, 2013). Al internalizar esta integración espacial, se comprende la interdependencia funcional, en particular el reconocimiento de que "lo que pasa en un lado afecta otros".

La ubicación y alcance espacial del valor parecen estar en función del tipo de valor y del significado que evoca. La asignación del valor de biodiversidad para toda la cuenca evidencia una atribución espacial más funcionalmente integrada e interdependiente donde se reconocen los vínculos socioecológicos. Por ejemplo, en general los participantes reconocían el rol importante de la biodiversidad en la parte alta (la más conservada), pero a su vez eran conscientes del papel trascendental de los ecosistemas de la cuenca media y baja para especies que migran de manera altitudinal. Otro factor que vincula el valor de la biodiversidad con toda el área de la cuenca es la actividad turística, pues se reconoce que el tipo de turismo que se desarrolla depende de la biodiversidad y riqueza ecológica que propicia la cuenca como sistema. En efecto, el valor social de la biodiversidad permite, en este caso, identificar en el participante patrones cognitivos que evidencian no sólo una valoración y un reconocimiento de la heterogeneidad ecosistémica a lo largo de la cuenca, sino también de la interdependencia funcional que implica esa diversidad. No se debe trivializar esta visión de interdependencia funcional, porque es muy probable que incida en los esfuerzos por articular acciones proambientales a nivel intercomunitario, así como en la creación de comités de cuenca que buscan una gestión más coordinada de los ecosistemas y poblaciones que dependen de éstos.

Por el contrario, los valores futuro, estético y de soporte de vida figuraron como los más asociados con el contexto espacial inmediato del participante (comunidad, localidad). Esto concuerda con estudios que reportan una relación estrecha entre ciertos valores sociales, como estética, recreación y espiritualidad, con sentido de apego a lugares especiales (Brown y Raymond, 2007; Raymond, Brown y Weber, 2010; Tuan, 1990). Es decir, el apego al lugar se asocia con una gama de valores que la persona relaciona con ese lugar en particular. Por ejemplo, el valor futuro, ${ }^{4}$ tal como fue definido en este estudio, vincula los

4 En algunos casos, al valor futuro se le llama también valor de opción o valor de legado. 
ecosistemas con el goce para futuras generaciones, por lo común las de parientes que viven (o vivirán) en el mismo lugar. El valor de soporte de vida está más asociado con el rol de los ecosistemas para procurar sustento y bienestar humano a través de funciones y procesos ecológicos. Sin embargo, si bien el valor de soporte de vida tiende a ser ubicado en el contexto local donde se reside, esto parece estar más en función de un mejor y más fácil acceso a estos servicios de soporte de vida y no tanto al lugar donde se dan esas funciones y procesos ecológicos. En otras palabras, se valora la obtención local de ese soporte de vida, indiferentemente de donde se den los procesos ecológicos que subyacen ese soporte.

Influencias en la espacialización

de los valores sociales en la cuenca del Savegre

Las transiciones económico-productivas y los usos de la tierra relacionados con ellas que se han dado en la cuenca desde la segunda mitad del siglo XX influyen de importantes maneras en los patrones espaciales de valores sociales en este sistema socioecológico. Estas transiciones, por ejemplo, explican en parte por qué se dan bajas intensidades de valores sociales en la cuenca baja, lo cual contrasta con el hecho de que los sistemas costeros se ubican entre los más ricos en valor y provisión de servicios ecosistémicos (Barbier, Hacker, Kennedy, Koch, Stier y Silliman, 2011; Costanza, Pérez-Maqueo, Martínez, Sutton, Anderson y Mulder, 2008; Groot, Brander, Ploeg, Costanza, Bernard, Braat, Christie, Crossman, Ghermandi, Hein, Hussain, Kumar, McVittie, Portela, Rodríguez, Brink y Beukering, 2012; Mendoza-González, Martínez, Lithgow, Pérez-Maqueo y Simonin, 2012).

La historia productiva contemporánea de la cuenca del Savegre se remonta más o menos a la primera mitad del siglo XX, cuando se establecen plantaciones bananeras en las inmediaciones de la actual ciudad de Quepos, a unos 20 kilómetros al oeste de la desembocadura del río Savegre. Los paisajes otrora poco alterados y con vestigios indígenas de inicios del siglo XX pasaron a ser sistemas de monocultivo. Como corolario, estas plantaciones fueron un factor de atracción para migrantes, tanto domésticos como internacionales, lo cual consolidó aún más la actividad productiva en la zona. No obstante, en la década de 1950, una serie de eventos hidrometeorológicos extremos afectó gran parte de las bananeras establecidas ahí, por lo que muchos productores desistieron de recuperar sus plantaciones. Así, inicia un declive gradual de la producción hasta 1985, cuando cierra de manera definitiva la compañía bananera establecida en la zona. En esa misma década, incursionan con más fuerza las plantaciones de palma africana, hoy muy prevalentes en la cuenca baja. En efecto, la mayor parte de las comunidades de este sector dependen hoy día de la actividad del cultivo de palma y en menor grado, del turismo. Por eso, esta transición relativamente temprana hacia una actividad agrícola de mayor intensificación y de monocultivos marcan, en gran medida, las concepciones espaciales de los valores sociales de servicios ecosistémicos en la cuenca del Savegre. 
Este auge de la producción de palma se dio de modo concomitante con el gradual, pero significativo, desarrollo del turismo de playa en asentamientos cercanos a la cuenca baja, como Quepos, Manuel Antonio y Dominical. En la cuenca baja del Savegre también han incursionado proyectos turísticos de mediana y pequeña escala en los últimos años, incluso en comunidades que desde hace bastante tiempo dependen de la palma. Estos proyectos turísticos se apoyan en una imagen "ecológica" o de "ruralidad" con el fin de atraer un perfil de turista que busca aventura, belleza escénica y estilo de vida simple.

Por otra parte, en la cuenca media predominó desde la década de 1960 la agricultura y ganadería de mediana y baja escala. Hoy en día destaca el cultivo del café, sobre todo en las zonas de transición hacia la cuenca alta. La incursión de éste modificó mucho el paisaje en este sector. Se estableció sobre todo en laderas con pendientes pronunciadas y por ende sujetas a procesos de sedimentación por una mayor exposición del suelo y con una mayor escorrentía superficial hacia cuerpos fluviales. El cultivo del café y su consolidación como principal actividad productiva en el país en general, y en la cuenca del Savegre en particular, se facilitaron en gran parte por flujos migratorios tanto internos como externos (Espinoza-Cisneros, 2018) que han hecho de comunidades como Los Ángeles, Santo Tomás y Santa Eduviges los principales focos productivos de la cuenca. En efecto, los valores sociales con carácter más “local”, como el valor futuro y el estético, son ubicados muy cerca de estos focos, debido a las mayores concentraciones de población que aquí se dan.

La cuenca alta es el sector con la topografía más pronunciada y abrupta, lo cual a lo largo de la historia ha indispuesto los asentamientos humanos a pesar de su cercanía al área metropolitana de Costa Rica. San Gerardo de Dota es quizás la comunidad más emblemática de este sector. Ésta ha pasado de depender, más o menos desde la década de 1960, de una producción agropecuaria y pesquera, a una economía basada en gran medida en el turismo y, en menor grado, en la agricultura. Al principio se establecieron colonizadores dedicados a la agricultura y a la ganadería, al cultivo de truchas y después al ecoturismo (Cruz, Chacón y Chacón, 2012). Es esta transición productiva hacia el turismo ecológico lo que subyace a mucha de la valoración social de los administradores en este sector, ya que con el advenimiento del ecoturismo en la cuenca se ha dimensionado mejor la gama de servicios que proveen los ecosistemas, incluso más allá de lo exclusivamente pecuniario. También percepciones de remotidad o inaccesibilidad de un sector de la cuenca son por lo común asociadas con ciertos valores sociales, como la biodiversidad y el aprendizaje. Aún muchas partes de la cuenca (sobre todo en los sectores de la cuenca alta y media) han tenido poca ocupación de actividades humanas, en parte porque están protegidas de manera legal pero también por su topografía abrupta.

Por eso, el éxito de la actividad ecoturística llevó a una valoración más exhaustiva del vínculo entre naturaleza y bienestar humano. No es de extrañar, entonces, que la biodiversidad haya sido el valor social más resaltado, al vincularse de forma directa no sólo con la razón que tiene el turista de visitar el lugar, sino también con un reconocimiento más explícito de la relación entre naturaleza y bienestar humano. Esta valoración más exhaustiva que ha 
propiciado el ecoturismo se ha proyectado incluso en comunidades donde esta actividad no es prominente. En general, en todas las comunidades estudiadas se percibió una fuerte noción de la importancia de la conservación y protección de los ecosistemas para garantizar la seguridad económica y la integridad ecológica en la región.

Las transiciones productivas y el uso de la tierra en la cuenca del Savegre también han influido en las apreciaciones del valor futuro. Por un lado, las crecientes presiones antropogénicas sobre los ecosistemas de la cuenca influyen en percepciones de vulnerabilidad socioecológica a futuro. Por otro lado, vinculado a lo anterior, la añoranza de los paisajes de antaño y del presente y los servicios ecosistémicos propician deseos de perpetuidad de los paisajes y de sus servicios. Como se dijo antes, una de las características destacables del valor futuro es su asociación con sentimientos de apego al lugar inmediato, en específico donde viven y se desenvuelven los productores, y cómo esto contrasta con el patrón visto para el valor de biodiversidad.

Las narrativas ambientales sobre la calidad del agua del río Savegre también inciden en las configuraciones espaciales de los valores sociales. No es inusual que se catalogue al río como el más limpio de América Latina o incluso del mundo. Esta narrativa es tan común en la concepción popular sobre la zona, que se menciona con frecuencia en medios nacionales y en sitios Web de promoción turística. Su origen no está claro, puesto que no hay estudios científicos que respalden esta afirmación. Consultas hechas a líderes comunitarios y gestores ambientales que trabajan en la cuenca tampoco revelaron el origen de esta narrativa. Sin embargo, se estima que la idea surge de un proyecto financiado por la Agencia de Cooperación Española a inicios de la década de 2000, bajo el programa llamado Araucaria (Gago García, 2000). Esa concepción de paisajes fluviales como algo limpio y poco alterado conlleva a una mayor atribución de ciertos valores sociales sobre servicios ecosistémicos, en especial por la poca presencia de humanos, la abundancia de agua y la amplia cobertura boscosa en la cuenca alta.

La cuenca del Savegre es muy vulnerable a desastres socio-naturales (Quesada-Román, Moncada-López, Paz-Tenorio, Espinoza-Jaime, Gutiérrez-Gutiérrez, Castellón-Meyrat y Acosta-Galeano, 2018; Saborío y Ureña, 2003). A lo largo de las últimas décadas, se han dado eventos hidrometeorológicos extremos y efectos relacionados con ellos, como deslizamientos de tierra, inundaciones y socavamientos de laderas adyacentes a los ríos que han causado daños considerables a las comunidades de la cuenca, y esto parece incidir en la valoración. Si bien no se contempló un valor social que capturara de modo más directo el servicio de protección contra eventos extremos, muchos participantes lo expresaron de forma indirecta a través de la biodiversidad y del valor futuro, ya que muchos están conscientes de la importancia de servicios ecosistémicos, como la fijación del suelo y retención del escurrimiento por parte de los bosques en laderas.

Por último, una influencia importante en la distribución espacial de los valores sociales atribuidos a los servicios ecosistémicos fue el ya mencionado proyecto Araucaria a inicios de la década de 2000 . Este proyecto, a través de sus 
actividades, buscó cambiar el modelo productivo en la cuenca, así como potenciar esfuerzos de conservación ambiental. Muchas iniciativas introdujeron diferentes alternativas de ingresos sin expandir la frontera agrícola y se buscaron opciones para mejorar encadenamientos productivos. A través de este proyecto se promovieron emprendimientos de turismo rural y se crearon y expandieron áreas protegidas dentro de la cuenca. Esto, a su vez, influyó en el imaginario sobre el valor tanto monetario como no monetario de los ecosistemas naturales y seminaturales de la cuenca. En efecto, los administradores de las unidades productivas, sobre todo los de mayor edad (de 50 años o más) mencionan el proyecto Araucaria como una iniciativa importante en los esfuerzos para mejorar la sostenibilidad dentro de la cuenca.

\section{Conclusiones}

Los factores cognitivos, antes relegados en evaluaciones ambientales, han ido adquiriendo mayor prominencia en campos disciplinarios sobre las relaciones entre el ser humano y el ambiente, sobre todo por el reconocimiento de que para comprender las decisiones y acciones ambientales a nivel individual e institucional es imposible soslayar los esquemas cognitivos. El ambiente y las cogniciones forman una relación de retroalimentación en la cual aquél influye en éstas y a su vez éstas determinan acciones sobre aquél.

En este artículo se examinaron las dimensiones espaciales de algunos valores sociales sobre servicios ecosistémicos en un microcosmos de retos de sostenibilidad comunes en el trópico global: la cuenca del río Savegre, Costa Rica. Se concluye que los valores sociales más priorizados se concentran en la zona de transición entre la cuenca alta y la cuenca media, donde se conjugan una alta fragilidad ecológica con los mayores focos de población y la mayor y más diversificada área productiva de la cuenca. Estos resultados también se analizan a la luz de las dinámicas socioecológicas en la cuenca desde mediados del siglo pasado, en especial las suscitadas en las últimas tres décadas.

Los resultados de la investigación confirman, entre otras cosas, el carácter más local de algunos valores sociales, como el valor futuro y estético, los cuales se asocian con el contexto espacial más inmediato del agente. Por otra parte, valores sociales como el de biodiversidad muestran, en este caso, tendencias hacia la generalización espacial bajo una visión de interdependencia funcional en el sistema de la cuenca. La posibilidad de incorporar estas representaciones espaciales de un área amplia más allá de un punto es, de hecho, un aspecto que hay que mejorar en herramientas tecnológicas SIGP, como SolVES.

Es relevante este tipo de análisis porque, primero, permite entender mejor las retroalimentaciones entre cogniciones y contextos socioecológicos. En este caso particular, se exploró la relación entre las dinámicas socioecológicas y los patrones espaciales de valoración social. Es importante recordar que esas valoraciones, a su vez, inciden de manera potencial en las conductas ambientales que modifican el espacio, lo cual crea una relación de retroalimentación. Segundo, porque refuerzan la necesidad de contemplar la gama de valores no 
monetarios atribuidos a los servicios ecosistémicos que aportarían de manera significativa a evaluaciones costo/beneficio más exhaustivas que trasciendan criterios estrictamente pecuniarios. Por último, el análisis de las dimensiones espaciales de los valores puede ser un insumo muy útil en procesos de planificación espacial y manejo sostenible de sistemas socioecológicos. Instrumentos de planificación que se alineen con la valoración social del espacio por parte de los pobladores, por ejemplo, tendrían más acogida, lo cual facilitaría la implementación de estrategias territoriales de sostenibilidad. En específico, se lograrían articular las preferencias de los pobladores con ciertas acciones de manejo, potenciando la ejecución e impacto de esas acciones. Así mismo estos insumos pueden robustecer de forma significativa ejercicios de zonificación, al incorporar criterios socioculturales explícitos en términos espaciales sobre las relaciones entre el ser humano y el ambiente. No obstante, para materializar estos aportes hay que continuar solidificando una visión integral y más exhaustiva en la gestión ambiental que entrelace de manera indivisible las dimensiones biofísicas con las sociales en pro de paisajes más sostenibles.

\section{Referencias}

Acevedo, H., Bustamante, J., Paniagua, L., y Chaves, R. (2002). Ecosistemas de la cuenca hidrográfica del Río Savegre, Costa Rica. Santo Domingo: Editorial INBio.

Arias-Arévalo, P., Martín-López, B., y Gómez-Baggethun, E. (2017). Exploring intrinsic, instrumental, and relational values for sustainable management of social-ecological systems. Ecology and Society, 22(4). doi: https://doi. org/10.5751/ES-09812-220443

Bagstad, K. J., Semmens, D. J., Ancona, Z. H., y Sherrouse, B. C. (2017). Evaluating alternative methods for biophysical and cultural ecosystem services hotspot mapping in natural resource planning. Landscape Ecology, 32(1), 77-97. doi: https://doi.org/10.1007/s10980-016-0430-6

Barbier, E. B., Hacker, S. D., Kennedy, C., Koch, E. W., Stier, A. C., y Silliman, B. R. (2011). The value of estuarine and coastal ecosystem services. Ecological Monographs, 81(2), 169-193.

Barrera Lobatón, S. (2009). Reflexiones sobre Sistemas de Información Geográfica Participativos (SIGP) y cartografía social. Cuadernos de Geografía: Revista Colombiana de Geografía, 18, 9-23.

Brondizio, E. S., y Gatzweiler, F. (2010). The socio-cultural context of ecosystem and biodiversity valuation. En P. Kumar (ed.), The Economics of Ecosystems and Biodiversity: Ecological and Economic Foundations (pp. 149-182). Londres: Earthscan.

Brown, G. (2013). The relationship between social values for ecosystem services and global land cover: an empirical analysis. Ecosystem Services, 5(0), 58-68. doi: https://doi.org/10.1016/j.ecoser.2013.06.004 
Brown, G., y Fagerholm, N. (2015). Empirical PPGIS/PGIS mapping of ecosystem services: a review and evaluation. Ecosystem Services, 13. doi: https://doi. org/10.1016/j.ecoser.2014.10.007

Brown, G., y Kyttä, M. (2014). Key issues and research priorities for public participation GIS (PPGIS): a synthesis based on empirical research. Applied Geography, 46, 122-136. doi: https://doi.org/10.1016/j.apgeog.2013.11.004

Brown, G., y Raymond, C. (2007). The relationship between place attachment and landscape values: toward mapping place attachment. Applied Geography, 27(2), 89-111. Recuperado de http://www.sciencedirect.com/science/ article/pii/S0143622806000464

Brown, G. , y Reed, P. (2012). Social landscape metrics: measures for understanding place values from public participation geographic information systems (PPGIS). Landscape Research, 37(1), 73-90.

Brown, G., Reed, P., y Harris, C. C. (2002). Testing a place-based theory for environmental evaluation: an Alaska case study. Applied Geography, 22(1), 49-76.

Bryan, B. A., Raymond, C. M., Crossman, N. D., y Macdonald, D. H. (2010). Targeting the management of ecosystem services based on social values: Where, what, and how? Landscape and Urban Planning, 97(2), 111-122. doi: https: //doi.org/10.1016/j.landurbplan.2010.05.002

Clement, J. M. (2006). Spatially Explicit Values on the Pike and San Isabel National Forests in Colorado (tesis doctoral). Colorado State University, Fort Collins, CO.

Codato, D. (2016). Estudio de la percepción social del territorio y de los servicios ecosistémicos en el Alto Mayo, Región San Martín, Perú. Espacio y Desarrollo, 31(27), 7-31. doi: https://doi.org/10.18800/espacioydesarrollo.201501.001

Costanza, R., Pérez-Maqueo, O., Martinez, M. L., Sutton, P., Anderson, S. J., y Mulder, K. (2008). The value of coastal wetlands for hurricane protection. Ambio, 37(4), 241-248. doi: https://doi.org/10.1579/0044-7447(2008)37[241:TVOCWF]2.0.CO;2

Cruz Conejo, L. D., Chacón Ureña, E., y Chacón Zúñiga, R. A. (2012). San Gerardo de Dota: en las montañas del Alto Savegre. San José, Costa Rica: Editorial MyF.

Dietz, T., Fitzgerald, A., y Shwom, R. (2005). Environmental values. Annual Review of Environment and Resources, 30, 335-372.

Espinoza-Cisneros, E. (2018). Optimizing social-ecological analysis of coupled human-river systems through the integration of conceptual frameworks: the case of the Savegre watershed, Costa Rica. Revista Geográfica de América Central, 61E(3), 32-50.

Espinoza-Cisneros, E., y Akhter, M. (2020). "Walking the talk" in land management : structural factors influencing pro-environmental intention-action links in a tropical watershed. Journal of Rural Studies, 79, 334-344. doi: https://doi.org/10.1016/j.jrurstud.2020.08.054

Gago García, C. (2000). Desarrollo sostenible en el medio tropical latinoamericano (el proyecto de desarrollo en la cuenca del río Savegre, Costa Rica). Anales de Geografía de la Universidad Complutense, 20, 253-264. 
García-Nieto, A. P., Quintas-Soriano, C., García-Llorente, M., Palomo, I., Montes, C., y Martín-López, B. (2015). Collaborative mapping of ecosystem services: the role of stakeholders' profiles. Ecosystem Services, 13. doi: https:// doi.org/10.1016/j.ecoser.2014.11.006

Gobierno de Costa Rica. (2015, 2 de julio). Gobierno decretó salvaguarda ambiental de 25 años para ríos Pacuare y Savegre. Comunicados de la Presidencia de la República de Costa Rica. Recuperado de http://presidencia.go.cr/comunicados/2015/08/gobierno-decreto-salvaguarda-ambiental-de-25-anos-para-rios-pacuare-y-savegre-2/

Groot, R. de, Brander, L., Ploeg, S. van der, Costanza, R., Bernard, F., Braat, L., Christie, M., Crossman, N., Ghermandi, A., Hein, L., Hussain, S., Kumar, P., McVittie, A., Portela, R., Rodríguez, L. C., Brink, P. ten, y Beukering, P. van (2012). Global estimates of the value of ecosystems and their services in monetary units. Ecosystem Services, 1(1), 50-61. doi: https://doi. org/10.1016/j.ecoser.2012.07.005

Intergovernmental Science-Policy Platform on Biodiversity and Ecosystem Services (IPBES). (2019). En E. S. Brondizio, J. Settele, S. Díaz, y H. T. Ngo (eds.), Global Assessment Report on Biodiversity and Ecosystem Services of the Intergovernmental Science-Policy Platform on Biodiversity and Ecosystem Services. Bonn: IPBES Secretariat.

Ives, C. D., y Kendal, D. (2014). The role of social values in the management of ecological systems. Journal of Environmental Management, 144, 67-72.

Jacobs, S., Dendoncker, N., Martín-López, B., Barton, D. N., Gomez-Baggethun, E., Boeraeve, F., McGrath, F. L., Vierikko, K., Geneletti, D., Sevecke, K. J., y others. (2016). A new valuation school: integrating diverse values of nature in resource and land use decisions. Ecosystem Services, 22, 213-220.

Johnson, D. N., Riper, C. J. van, Chu, M., y Winkler-Schor, S. (2019). Comparing the social values of ecosystem services in US and Australian marine protected areas. Ecosystem Services, 37(marzo), 100919. doi: https://doi. org/10.1016/j.ecoser.2019.100919

Jones, N., Shaw, S., Ross, H., Witt, K., y Pinner, B. (2016). The study of human values in understanding and managing social-ecological systems. Ecology and Society, 21(1).

Larson, K. L. (2010). An integrated theoretical approach to understanding the sociocultural basis of multidimensional environmental attitudes. Society and Natural Resources, 23(9), 898-907.

Martín-López, B., Gómez-Baggethun, E., García-Llorente, M., y Montes, C. (2014). Trade-offs across value-domains in ecosystem services assessment. Ecological Indicators, 37, 220-228.

Mattey, J. (2014). El proyecto desarrollo sostenible de la cuenca hidrográfica del río Savegre, una experiencia de desarrollo rural territorial. Ministerio de Agricultura y Ganadería (MAG)-Oficina Quepos, Costa Rica.

Mendoza-González, G., Martínez, M. L., Lithgow, D., Pérez-Maqueo, O., y Simonin, P. (2012). Land use change and its effects on the value of ecosystem services along the coast of the Gulf of Mexico. Ecological Economics, 82, 23-32. 
Meyfroidt, P. (2013). Environmental cognitions, land change, and social-ecological feedbacks: an overview. Journal of Land Use Science, 8(3), 341-367. doi: https: / / doi.org/10.1080/1747423X.2012.667452

Millennium Ecosystem Assessment (MEA). (2005). Ecosystems and human well-being: current state and trends. Vol. 1. Whashington D.C.:Island Press.

Ministerio de Ambiente y Energía (MINAE). (2004). Plan de Manejo Integrado de la Cuenca Hidrográfica del Río Savegre. Programa Araucaria, Costa Rica.

Naranjo, B. (2016). Ecología trófica de la trucha arcoíris Oncorhynchus mykiss (Salmonidae) en el río Savegre, San Gerardo de Dota, Costa Rica (tesis de licenciatura). Escuela de Biología, Universidad de Costa Rica.

Nassauer, J. (1995). Culture and changing landscape structure. Landscape Ecology, 10(4), 229-237.

Potschin-Young, M., Haines-Young, R., Görg, C., Heink, U., Jax, K., y Schleyer, C. (2018). Understanding the role of conceptual frameworks: reading the ecosystem service cascade. Ecosystem Services, 29, 428-440.

Quesada-Román, A., Moncada-López, R., Paz-Tenorio, J. A., Espinoza-Jaime, E., Gutiérrez-Gutiérrez, C., Castellón-Meyrat, A., y Acosta-Galeano, N. (2018). Las investigaciones sobre movimientos de laderas en Costa Rica, Honduras, México y Nicaragua: enseñanzas desde la academia, las agencias de cooperación y las instituciones públicas. Revista Geográfica de América Central, 1(60), 17. doi: https://doi.org/10.15359/rgac.60-1.1

Raymond, C. M., Brown, G., y Weber, D. (2010). The measurement of place attachment: personal, community, and environmental connections. Journal of Environmental Psychology, 30(4), 422-434. Recuperado de http://www. sciencedirect.com/science/article/ pii/S0272494410000794

Riper, C. J. van, y Kyle, G. T. (2014). Capturing multiple values of ecosystem services shaped by environmental worldviews: a spatial analysis. Journal of Environmental Management, 145, 374-384.

Riper, C. J. van, Kyle, G. T., Sutton, S. G., Barnes, M., y Sherrouse, B. C. (2012). Mapping outdoor recreationists' perceived social values for ecosystem services at Hinchinbrook Island National Park, Australia. Applied Geography, 35(1-2), 164-173.

Saborío, J., y Ureña, M. (2003). Estudio riesgo integral en la cuenca del río Savegre. Instituto Costarricense de Electricidad (ICE), Costa Rica.

Sherrouse, B. C., Clement, J. M., y Semmens, D. J. (2011). A GIS application for assessing, mapping, and quantifying the social values of ecosystem services. Applied Geography, 31(2), 748-760. doi: https://doi.org/10.1016/j. apgeog.2010.08.002

Sherrouse, B. C., y Semmens, D. J. (2015). Social Values for Ecosystem Services, Version 3.0 (SolVES 3.0) -Documentation and User Manual. United States Geological Survey (USGS).

Sherrouse, B. C., Semmens, D. J., y Clement, J. M. (2014). An application of social values for ecosystem services (SolVES) to three national forests in Colorado and Wyoming. Ecological Indicators, 36, 68-79. doi: https://doi. org/10.1016/j.ecolind.2013.07.008 
Sistema Nacional de Áreas de Conservación (SINAC). (2017). Sistematización desarrollo sostenible de la cuenca hidrográfica del río Savegre. Sistema Nacional de Áreas de Conservación, Ministerio de Ambiente y Energía de Costa Rica.

Sun, F., Xiang, J., Tao, Y., Tong, C., y Che, Y. (2019). Mapping the social values for ecosystem services in urban green spaces: integrating a visitor-employed photography method into SolVES. Urban Forestry \& Urban Greening, 38, 105-113. doi: https://doi.org/10.1016/j.ufug.2018.11.012

Tadaki, M., Sinner, J., y Chan, K. M. A. (2017). Making sense of environmental values: a typology of concepts. Ecology and Society, 22(1). doi: https://doi. org/10.5751/ES-08999-220107

Tuan, Y.-F. (1990). Topophilia: A Study of Environmental Perceptions, Attitudes, and Values. New York: Columbia University Press.

United Nations Educational, Scientific and Cultural Organization (UNESCO). (2017). Savegre Biosphere Reserve. En United Nations Educational, Scientific and Cultural Organization. Recuperado de http: / /www.unesco.org/new/ en/natural-sciences/environment/ecological-sciences/biosphere-reserves/ latin-america-and-the-caribbean/costa-rica/savegre/

Vila Subirós, J., Linde, D. V., Llausàs Pascual, Al., y Ribas Palom, A. (2006). Conceptos y métodos fundamentales en ecología del paisaje (landscape ecology). Documents d'anàlisi Geogràfica, 151-166.

Vreese, R. De, Leys, M., Fontaine, C. M., y Dendoncker, N. (2016). Social mapping of perceived ecosystem services supply -the role of social landscape metrics and social hotspots for integrated ecosystem services assessment, landscape planning and management. Ecological Indicators, 66, 517-533. doi: https: / / doi.org/10.1016/j.ecolind.2016.01.048

West, S., Haider, L. J., Masterson, V., Enqvist, J. P., Svedin, U., y Tengö, M. (2018). Stewardship, care and relational values. Current Opinion in Environmental Sustainability 35, 30-38. doi: https://doi.org/10.1016/j.cosust.2018.10.008 